\title{
Le brouillage des frontières de la société salariale dans les Amériques et au-delà : une lecture des transformations du travail dans un globalising world
}

Blurring of Wage-Earning Society Borders in the Americas and Beyond: A

Reading of the Labour Transformations in a Globalising World.

Lo confuso de las fronteras de la sociedad salarial en las Américas y más allá:

una lectura de las transformaciones del trabajo en un globalising mundo.

\section{Christian Azaïs}

\section{OpenEdition}

\section{Journals}

Édition électronique

URL : https://journals.openedition.org/ideas/872

DOI : $10.4000 /$ ideas.872

ISSN : 1950-5701

Éditeur

Institut des Amériques

Référence électronique

Christian Azaïs, «Le brouillage des frontières de la société salariale dans les Amériques et au-delà une lecture des transformations du travail dans un globalising world », IdeAs [En ligne], 5 | 2015, mis en ligne le 21 mai 2015, consulté le 19 octobre 2022. URL : http://journals.openedition.org/ideas/872 ; DOl : https://doi.org/10.4000/ideas.872

Ce document a été généré automatiquement le 19 octobre 2022.

Creative Commons - Attribution - Pas d'Utilisation Commerciale - Pas de Modification 4.0 International - CC BY-NC-ND 4.0

https://creativecommons.org/licenses/by-nc-nd/4.0/ 


\title{
Le brouillage des frontières de la société salariale dans les Amériques et au-delà : une lecture des transformations du travail dans un globalising world
}

\author{
Blurring of Wage-Earning Society Borders in the Americas and Beyond: A \\ Reading of the Labour Transformations in a Globalising World. \\ Lo confuso de las fronteras de la sociedad salarial en las Américas y más allá: \\ una lectura de las transformaciones del trabajo en un globalising mundo.
}

Christian Azaïs

\section{Introduction}

1 Deux voies sont possibles pour analyser les transformations et la crise du travail aujourd'hui ${ }^{1}$. L'on peut considérer qu'elles sont le fruit des contradictions internes du capitalisme et renvoient à ses dérives financières ou alors y déceler une exacerbation de ce qui se passait déjà dans les sociétés salariales et considérer alors que rien de bien nouveau ne s'est produit. Peut-on, toutefois, les résumer à une amplification de processus déjà en cours ou faut-il constater, dans cette crise, un changement de nature? Telle est l'interrogation générale de ce texte.

Le thème de la crise du travail n'est pas nouveau en soi. Déjà en 1997, après la parution des ouvrages sur la «fin du travail » (Méda D., 1995 ; Rifkin J., 1996), la question «Quelle crise? » à propos du travail était posée (Eckert H., 1997). À cette occasion, les auteurs signalent six facettes de la crise du travail :

L'extension d'un chômage de masse; la précarisation de la relation salariale; le

déni des capacités mobilisées dans l'entreprise ; la difficulté à répondre à l'exigence 
de sens; l'affaiblissement des acteurs collectifs traditionnels de la relation de

travail et enfin la crise des modes de connaissance du travail ${ }^{2}$.

Fort de ces considérations qui demeurent d'actualité, auxquelles se greffe l'individualisation de la relation salariale, je me propose d'envisager la crise du travail sous l'angle de la globalisation, d'une globalisation en cours, non uniforme, et de ses effets. Ce terme est préféré à celui de mondialisation car il en souligne les deux processus constitutifs, l'uniformisation et la différenciation, qui traitent d'une évolution des formes de mise au travail caractérisée par le brouillage des frontières du salariat, aussi bien au Nord qu'au Sud. Ce flou des frontières du salariat est cohérent avec le concept d'hybridation, qui traduit l'enchevêtrement des formes de mise au travail et de contrats de travail. Le binôme flexibilité/précarité le caractérise mais pas uniquement, puisque depuis plusieurs années la flexibilité n'est pas que l'apanage des travailleurs $\mathrm{du} \mathrm{Sud}^{3}$ tout comme la précarité ne concerne pas uniquement les travailleurs pas ou peu qualifiés. Ces processus illustrent les transformations des marchés du travail contemporains. Ce texte pose des jalons théoriques pour comprendre le bouleversement des frontières des formes de mise au travail, à partir de dimensions temporelles et spatiales.

\section{Vers un globalising world?}

$4 \quad$ L'idée d'un globalising world ${ }^{4}$ emprunte davantage à la science politique et à la sociologie qu'à l'économie, qui lui préfère dans sa version française le concept de mondialisation. La mondialisation n'est que l'expression de la concurrence et de l'extension des marchés; ses multiples facettes sont passées sous silence, notamment son contenu éminemment politique (Azaïs C., 2010a:10), souligné auparavant par David Held et Anthony McGrew (Held D., McGrew A., 2000). Comme le soutient à juste titre Giraud (Giraud O., 2012), la globalisation n'est pas qu'une conséquence de la concurrence, elle influe sur différents niveaux sociétaux. Elle met en scène une pluralité d'interactions, sur des plans aussi divers que les sphères locale, globale, culturelle et politique. Elle n'en oublie pas pour autant la sphère économique : «C'est une force, une dynamique qui, sans supplanter celles déjà existantes, vient tordre, bouleverser et métamorphoser les équilibres antérieurs. Elle produit des effets semblables sur les acteurs, les institutions, les marchés, les logiques sociales, etc.» (Giraud 0., 2007 : 389). Définie comme une suite d'interdépendances multiples de phénomènes sociaux, économiques et politiques, la globalisation renvoie à deux mouvements concomitants, la standardisation et la différenciation. Cette dernière prime sur la standardisation et s'impose à deux niveaux : la mise en concurrence des individus entre eux ainsi qu'entre les lieux où ils exercent leur activité. Ainsi, l'extension à l'échelle planétaire de certaines formes de salarisation et de mise au travail apparaît-elle comme un facteur d'homogénéisation que des appropriations nationales ou locales différencieront, les deux processus n'étant pas contradictoires. La globalisation n'est donc pas uniquement homogénéisante. Elle scande ce que d'aucuns ont qualifié de «tectonique des territoires" (Lacour C., 1996), qui souligne la prégnance des déterminations temporelles et spatiales dans la construction territoriale pour comprendre les formes de mise au travail. 


\section{Le brouillage des temps et des espaces : son impact sur le travail}

5 En tant qu'" activité productive organisée socialement » (Eckert H., $1997: 54$ ), le travail est nécessairement en contact avec le reste de la vie: le relier à la globalisation lui confère une grandeur de fait social total et l'associe aux événements qui marquent les sociétés, notamment les crises. Dans le décryptage de la globalisation, les dimensions temporelle et spatiale sont indissociables.

6 La dimension temporelle se décline en deux mouvements : l'un combine le temps long du long terme et le temps court du court terme; l'autre, le temps lent et le temps rapide, qui renvoient à la qualité de l'objet étudié. Le temps long est en lien avec l'histoire des nations, c'est le temps historique de Braudel; le temps court rappelle le temps des " expériences $\|^{5}$ des individus. Pour sa part, le temps lent renvoie à l'histoire des sociétés pré-industrielles, admirablement décrites par Thompson (Thompson E. P., 1979) ; il s'adresse aussi au temps bricolé des interstices du travail... et de la vie ; « on bricole l'emploi comme on bricole sa vie » (Hatzfeld H., Hatzfeld M., Ringart M., 1998). Jongler avec un temps rapide et un temps court a toujours été l'apanage des travailleurs informels qui, à peine le ciel obscurci à l'horizon, proposent déjà au chaland une panoplie de parapluies posés sur un étal de fortune prestement improvisé sur un trottoir, au coin d'une rue ou à la sortie d'un commerce, d'un lieu public ou d'un shopping center. À l'heure actuelle, dans les sociétés développées, une telle situation est le lot d'individus précarisés en quête de survie pour pallier la perte de leur emploi ou les conséquences d'un temps partiel subi - entraînant un salaire insuffisant pour vivre.

7 Lire le travail et l'emploi à partir de la dimension temporelle présente l'intérêt de ne pas se limiter à une partition catégorique dans laquelle le travail au Nord relèverait d'un type de temporalité et celui au Sud d'une autre. Ces deux rythmes de temps s'enchevêtrent. Le temps permet aussi d'apprécier l'imbrication entre activités formelles et informelles, l'informel s'immisçant dans toutes les sphères de l'activité humaine, ce qui méthodologiquement invalide toute appréhension dichotomique des faits ${ }^{6}$.

8 Appréhender le sous-développement, en faisant donc implicitement référence au Sud, revient à penser au bricolage entre un temps long et un temps court, tout autant qu'au jonglage entre un temps lent et un temps rapide. Le bricolage entre le temps long et le temps court permet de ne pas perdre de vue la temporalité longue dans laquelle s'inscrivent les événements d'aujourd'hui, celle, par exemple de sociétés marquées par une colonisation qui transparaît dans leur identité. De même, la superposition entre temps lent et rapide s'applique aux deux univers, développés et en développement. Il n'y a pas un temps lent qui serait celui des sociétés en développement au Sud et un temps rapide propre au monde développé au Nord. L'imbrication des deux types de temps représente une tendance lourde de nos jours.

9 L'interpénétration des formes temporelles évoquées a une incidence sur la compréhension de la complexité des situations de travail et d'emploi. Le travail incarne la pluralité des temporalités. Dans sa version moderne, il est «mode d'activité caractérisable par la recherche d'un résultat dans le moindre temps », il s'inscrit dans une logique d'« économie du temps» (Bidet J., 1994:245) et se rapporte alors à la mobilisation de deux types de temps, un temps court, celui de l'immédiateté et un 
temps rapide, celui de "l'économie du temps ", c'est-à-dire de l'adaptation rendue nécessaire par le rythme des transformations. Les entreprises sont tenues entre, d'un côté, la nécessité de réduire les coûts pour rester compétitives (elles font alors jouer au salaire le rôle de variable d'ajustement et utilisent la flexibilité contractuelle) et, de l'autre, l'impératif de maintenir en leur sein un volet fixe de travailleurs, capables de porter le projet entrepreneurial et de servir de mémoire de l'entreprise. Or, aujourd'hui, le propre de l'emploi pour un individu est d'avoir de plus en plus de mal à s'inscrire dans la continuité, soit parce qu'il est précaire et éphémère, soit parce que sa trajectoire de travail est davantage vulnérable aux aléas de la conjoncture. En cela il se rapproche des travailleurs informels du Sud dont le cycle de travail oscille fréquemment entre périodes d'inactivité ou d'emploi dans des activités formelles et informelles. Toutefois, le niveau de protection sociale et la présence plus ou moins prégnante d'un État providence s'imposent toujours comme trait majeur de différenciation entre travailleurs du Nord et du Sud. Les formes de mise au travail et du rapport salarial ont évolué. Peut-on pour autant en déduire qu'elles sont identiques au Nord et au Sud, conformément à ce que laisserait supposer une acceptation unilatérale $\mathrm{du}$ brouillage des frontières salariales ou de l'hybridation? L'importance de la dimension spatiale apparaît alors, c'est elle qui permet de faire le lien entre le travail et la dimension temporelle. En effet, les rapports de travail obéissent à un temps d'apparition facilement assimilable à un temps court, mais ils ne sont pas moins exempts de l'influence de leurs ancrages territoriaux qui leur confèrent un lien avec le passé des territoires, construit sur la base des « expériences » des individus.

L'articulation entre le travail et la dimension spatiale se fait par la temporalité : une temporalité courte qui a besoin de la temporalité longue du territoire pour s'exprimer pleinement et s'ancrer spatialement dans la mesure où le travail façonne le territoire (la mobilité des individus, leurs expériences individuelles et collectives en sont le témoin). Il s'ensuit que les perturbations que connaît un territoire (suite à une délocalisation, au rachat d'une entreprise par une autre, à la décision d'actionnaires d'arrêter ou au contraire de renforcer la production sur un site) ont forcément un impact sur le travail et sur la forme d'insertion des individus sur le marché du travail. Ainsi, le travail incarne-t-il de plus en plus la diversité spatiale.

Le brouillage des deux dimensions temporelle et spatiale peut conduire à une perte des repères que la crise accentue peut-être, mais qui est inhérente à l'évolution contemporaine des sociétés. D'un point de vue théorique, il permet de questionner des catégories incapables de rendre compte de la réalité. La notion d'hybridation aide à mieux appréhender ces phénomènes difficilement saisissables en raison de l'enchevêtrement de formes nouvelles de mise au travail, appuyées sur les expériences d'engagement individuel. Reste à en élaborer le cadre juridique. Pour l'heure, c'est le règne du flou des frontières entre situations de travail protégées et non protégées. Tout un continuum de situations compose l'espace dévoilé par cette dichotomie, réconfortante peut-être pour l'esprit mais peu opérationnelle. La notion d'hybridation est censée rendre compte de ces brèches laissées sans interprétation. 


\section{Dé-segmentation et hybridation : l'institutionnalisation du flou}

12 La «zone grise » (Supiot A., 2000) de cet entre-deux illustre la panoplie de formes de mise au travail que les figures normatives de "dépendant» ou " autonome » ne sont plus à même de qualifier, la relation d'emploi ne se limitant pas à cette dichotomie (Azaïs C., 2010b). Seul le recours à la jurisprudence peut aider à alimenter une science juridique mal à l'aise avec la prolifération de contrats de travail (Accornero A., 1998 ; Antonmattei P.-H., Sciberras J.-C. ; 2008), pour ne pas mentionner les réalités qui échappent de plus en plus au cadre réglementaire.

Pendant un temps, j'avais avancé l'idée d'une dé-segmentation du marché du travail (Azaïs C., 2006), pour signifier un certain brouillage des frontières entre les formes de mise au travail et le fait que l'on aurait affaire à des situations de moins en moins scandées par, d'un côté, des formes protégées et, d'un autre, des formes non protégées. Cette acception a été abandonnée car l'idée de dé-segmentation pouvait faire croire à un univers sans aspérité, sans antagonisme et sans conflit, donc incompatible avec l'idée de différenciation. En fait, un mouvement contradictoire de rigidification et de flexibilisation se produit sur le marché du travail. Paradoxalement, la notion de « zone grise » (Supiot A., 2000), de « flou » (Azaïs C., 2010c) semble plus précise pour traiter des frontières du salariat, car l'idée de frontière, en français, débouche sur l'hypothèse de statuts tranchés différenciant les travailleurs entre eux (au même titre que le terme anglais border), alors que l'acception anglaise de boundaries est plus à même de rendre compte de la zone intermédiaire entre deux situations. Or, les spécialistes s'accordent sur le fait que nombre de travailleurs se situent dans une zone entre le travail salarié et le travail indépendant. Il est ainsi question de «travailleur économiquement dépendant» (Antonmattei P.-H., Sciberras J.-C., 2008). Cette qualité a trouvé des adeptes auprès des décideurs politiques, qui ont mis en place des dispositifs en Allemagne, Espagne, Grande-Bretagne et Italie visant à entériner et encadrer des relations d'emploi nouvelles a posteriori. Grignotage de principes liés à la protection du travailleur salarié, d'une part, reconnaissance de la spécificité du travailleur économiquement dépendant, d'autre part, témoignent des frontières mouvantes du salariat, ce que désigne l'hybridation ou l'institutionnalisation du flou, dans la mesure où la flexibilité, la multiplication des statuts pour une même profession, la précarité et l'informel deviennent la norme. Toutefois, l'hybridation s'accommode parfaitement d'une montée de la salarisation, phénomène perceptible dans des sociétés aussi différentes que la française ou la brésilienne.

L'hybridation se révèle alors être l'un des outils analytiques reflétant les mutations du travail et de l'emploi. Elle évoque l'émergence de phénomènes apparemment semblables au Sud et au Nord et permet de comparer des situations, sans pour autant gommer les spécificités nationales. Dans cette perspective, elle se différencie de l'acception selon laquelle le Sud servirait de modèle d'analyse au Nord, elle aide même à saisir des tendances qui vont en sens inverse et dès lors participe de l'entendement de la globalisation, celle-ci jouant sur les spécificités des États-nations. Par exemple, si les attaques contre les garanties et les droits sociaux sont monnaie courante, se ressemblent et touchent les pays développés et les moins développés, rien ne permet de conclure à une uniformité des situations repérées. La différenciation vient alors tempérer l'uniformisation. L'hybridation prend en compte à la fois l'existence et le 
bouleversement de nouveaux contours des situations de travail et d'emploi. La nature intrinsèquement conflictuelle du travail lui confère une dynamique qui est à l'origine de l'état de crise permanent qui le caractérise. Les spécificités temporelles et spatiales confortent l'assertion selon laquelle les analyses ne sont ni transposables ni comparables stricto sensu. Flou, brouillage, bricolage sont les diverses facettes de normes relatives au travail et à la protection sociale qui mettent en lice des rapports de forces mouvants. Savoir si elles seront institutionnalisées ou pas ne peut se décréter a priori.

\section{Les contours de la relation salariale : une différenciation croissante}

La relation salariale est le théâtre de nouvelles frontières, lieux de disputes autour de l'appropriation des richesses. Ceci s'apparente à la "crise du travail», qui tient davantage à une crise du partage des richesses et de l'appropriation. Les données sur l'évolution récente du partage de la valeur ajoutée en France l'attestent (Insee, 2009).

La stabilité du partage repérée dans l'hexagone occulte un changement dans la structure interne du marché de l'emploi, en raison de la salarisation croissante et des divers statuts qu'elle dévoile. Le salaire net par tête augmente, bien que lentement, mais de façon nettement différenciée. Le taux annuel de croissance moyenne du salaire net est de $1 \%$ sur la période 1998-2005 pour le décile P090-P100, il est de 6,1\% pour le $99^{e}$ centile (+50\%). Le salaire net du décile le moins bien loti (P0-P090) n'a progressé que de $0,4 \%{ }^{7}$. La stabilité salariale générale dévoile donc une profonde différenciation, fruit de la discontinuité des trajectoires professionnelles d'individus qui naviguent entre situations d'emploi, de chômage et d'inactivité. Autant d'éléments qui corroborent la thèse de l'hybridation.

La tendance à l'accroissement des différenciations entre salariés n'est pas une exception française. Elle se vérifie dans la plupart des pays du Nord qui ont connu, au cours de la même période, une chute de la part des salaires dans la valeur ajoutée par rapport au PIB (-8,9 points de pourcentage en Italie ; - 7 au Canada ; - 6,6 en France ; 6,2 aux Etats-Unis ; - 5,3 au Japon et - 4,8 points au Royaume-Uni). Sur la seule période 2001/2007 ce mouvement s'amplifie : - 5,6 points au Japon ; - 4,1 points en Allemagne ; parfois plus lentement :-0,8 au Canada; - 0,6 en France (Commission européenne, 2010).

18 Au Brésil, par exemple, en 1990, la part du travail dans le revenu national était de $45,4 \%$. Elle n'était plus que de $41,7 \%$ en 2007. Malgré l'augmentation sensible du salaire minimum ces dernières années et la diminution du nombre total de pauvres, l'écart entre les plus riches et les plus pauvres s'est creusé en 2012 (PNAD/IBGE, 2013). L'enquête révèle que le $1 \%$ des plus riches a davantage progressé, en 2012, que les $10 \%$ les plus pauvres, contrairement aux années antérieures. En 2012, le revenu du travail a augmenté de $10,8 \%$ pour les plus riches et seulement de $6,6 \%$ pour les plus pauvres. Leur part dans le total des revenus n'a pas bougé $(1,4 \%)$, celle des plus riches a grimpé de $12 \%$ en 2011 à 12,5\% en 2012. Si l'on ajoute aux revenus du travail ceux provenant des loyers, retraites, transferts de revenus et des placements financiers, la croissance du revenu des plus pauvres n'a été que de $5,1 \%$ contre $12,8 \%$ pour les plus riches. L'augmentation moyenne atteint $5,6 \%^{8}$. La distribution fonctionnelle du revenu n'a pas 
été favorable aux travailleurs brésiliens. Ces données dévoilent le caractère erratique et mouvant du partage de la richesse et de la lecture que l'on peut en faire.

Un temps s'est posée la question d'un Sud, laboratoire préfigurant l'éclosion de situations identiques au Nord. Aujourd'hui, comme par effet de boomerang, les transformations au Nord sont en passe de concerner le Sud. Pour exemple, dans le champ de la protection sociale, de plus en plus d'entreprises tendent à imiter leurs consœurs étatsuniennes et tendent à se substituer à l'État dans l'octroi de benefits (Cappellin P., Giffoni R., 2007).

Ainsi, si dans les années 1960-1970 le discours en Amérique latine était axé sur un accroissement de la salarisation et un recul des activités informelles en raison de la progression de l'industrialisation et d'un exode rural moins intense, la réalité a très vite déjoué les prédictions. L'expansion des activités informelles a gagné tous les pays, ce qui ne les a pas empêchés de connaître simultanément une extension du salariat, sans toutefois que la protection sociale publique reliée à une notion de salariat reçue en Europe ne suive.

21 Ensuite, faisant référence à la multi-activité à laquelle doivent désormais se soumettre les travailleurs, Beck défendra la «brésilianisation» de l'Occident (Beck Ü., 2000). Il met l'accent sur l'une des caractéristiques centrales de l'évolution de certaines professions et de certains marchés du travail. La multi-activité serait dorénavant la marque de fabrique de sociétés où l'emploi attrayant, hautement qualifié, à plein temps et bien payé ne serait que chose du passé9. C'est ne pas prendre en compte la spécificité de la construction de chaque formation sociale; c'est aussi aller à l'encontre de ce que l'exemple français a montré.

Dans de nombreux pays l'augmentation de la salarisation, censée représenter une amélioration pour les travailleurs (tout du moins dans l'imaginaire collectif) s'est accompagnée d'une atteinte à leurs droits sociaux et d'un accroissement des inégalités, dévoilant des formes de mise au travail de plus en plus poreuses.

Le travail salarié a été présenté comme un universel que l'on devrait retrouver partout ${ }^{10}$, assertion hautement normative et ethnocentrique, qui passe sous silence tout un pan de formes de mise au travail relevant de logiques qui s'éloignent de l'idéal-type du travail salarié. Une telle attitude revient à se priver de la compréhension du foisonnement de situations de travail, à laquelle avaient habitué les analyses sur le travail informel (Lautier B., De Miras C., Morice A., 1991). Dobb avait déjà souligné la difficulté de théoriser à partir de situations de travail concrètes, en raison des « complexités du monde réel » et de la multiplicité des situations de travail (Maurice Dobb, $\left.1955: 89^{11}\right)$.

24 À cette difficulté de théorisation sur les salaires s'ajoute celle de la comparaison. La déstabilisation des normes d'emploi perceptible sur le marché du travail confère une certaine acuité à la différenciation et à la standardisation, imbriquées de manière indissociable dans la globalisation. Les frontières entre situations du travail formelles et informelles, protégées et non protégées tendent à voler en éclat. Les professions qui a priori devraient être les plus à l'écart des bouleversements, comme celles qui requièrent des qualifications spécifiques et relèvent de marchés du travail quasiment fermés ne sont pas épargnées par la flexibilité, mais une flexibilité qui n'est pas synonyme de précarité (Azaïs Ch., 2010c). La seule uniformisation, s'il en est, porte sur l'apparition d'une zone grise dont les contours imprécis questionnent le salariat. Il ne reste plus qu'à la qualifier. L'émergence de configurations nouvelles de mise au travail, 
rappelant à certains égards des formes anciennes, ne saurait être épuisée par une lecture avec les outils habituels, en partie en raison de l'impact de la globalisation. La difficulté réside désormais dans la construction de nouvelles catégories analytiques pour saisir la particularité du travail. Tel est le bienfait de la crise du travail.

\section{Conclusion}

En temps de crise l'état normal est celui de l'incertitude et du déséquilibre, «le long fleuve tranquille " étant anomalie. Rapportée au travail, la situation de non crise est l'exception : elle est a-normale et se traduit par une perte ou une absence de normes, mettant à jour des situations floues. Un temps épargnés ou moins affectés, grâce à la présence d'un État-providence puissant, les pays développés sont confrontés à ce qui faisait la spécificité des sociétés en développement avec leur cortège d'activités informelles et de situations difficilement définissables. L'inconnue tient à la nature de cette crise : marque-t-elle un changement de régime d'accumulation ou n'est-elle que la suite d'un mouvement inhérent à l'histoire du travail et de ses luttes? À mon sens, il s'agit d'un tournant, qui reflète la tendance à l'individualisation de la relation salariale et fait de plus en plus du salaire une variable d'ajustement ${ }^{12}$.

La globalisation et son impact sur le travail sont au cœur de la question théorique de cet écrit. Pour saisir les deux processus constitutifs de la globalisation, l'uniformisation et la différenciation, l'accent a été mis sur la prégnance des dimensions temporelles et spatiales, car elles donnent la mesure des compositions sociales et politiques (flexibles, contingentes, labiles) des formes de mise au travail.

L'uniformisation et la différenciation ne laissent présager d'aucune configuration unique pour les marchés du travail contemporains, tout dépend du mode d'insertion des pays dans la division internationale du travail. Tout au plus peut-on entrevoir des mouvements, aisément réversibles, relevant d'une précarisation qui prend la forme d'une atteinte aux droits sociaux et à la protection sociale. Elle s'exprime par le biais d'une réorientation des politiques sociales, dorénavant davantage tournées vers l'assistance à des populations ciblées (femmes pauvres, minorités ethniques) que visant à l'universalisation, le Brésil s'en est fait le champion : le programme Bolsa familia, par exemple, l'atteste ${ }^{13}$. Aux femmes pauvres traditionnellement cantonnées au rôle de reproduction sociale, le gouvernement fait jouer maintenant un rôle important dans la production sociale, en leur offrant des emplois, mal payés, pas suffisamment pour faire vivre leur famille, alors que les hommes pauvres, eux, sont exclus du marché du travail et ne vivent que d'expédients (Destremau B., Georges I., 2015). Parallèlement à ce mouvement, il n'est pas rare de voir des entreprises (des grandes) se substituer à l'État en matière de couverture sociale, ce qui, à une époque où le chômage croît dans nombre de pays, n'augure en rien d'une amélioration future pour des millions de personnes laissées pour compte ou...devant travailler à leur compte.

La mobilisation des individus vers le salariat, puis leur fixation dans le salariat et enfin leur mobilité en son sein a constitué l'histoire du salariat dans les pays développés. Cette séquence est aujourd'hui mise à mal, en partie seulement, dans la mesure où le salariat progresse, même dans des pays en développement. Cependant, sa nature a évolué. Pour en pointer les bouleversements, je me suis attelé à souligner les frontières du salariat et à essayer de comprendre la zone grise du salariat, cette zone interstitielle qui rend caduques les dichotomies (davantage pensées par les chercheurs que 
présentes dans la réalité) telles que travail formel/travail informel; travail/horstravail ; qualification/protection sociale.

Aujourd'hui, les droits sociaux, la protection sociale, les garanties assorties à l'exercice d'une profession sont affectés et requièrent une autre approche pour analyser le travail et le salariat, car celle qui prend l'idéal-type fordien comme modèle ou paramètre analytique évince les spécificités. C'est en cela que consiste la « crise du travail ». Peutêtre plus que jamais, il est difficile de distinguer les contours du salariat et de délimiter les situations de dépendance de celles d'autonomie au travail. Les frontières du salariat ont tendance à s'élargir, à se décloisonner, ce qui remet en cause nombre de catégories jusque-là considérées comme centrales (et immuables?) relatives au travail et au salariat. Un vaste chantier s'ouvre. Autant de questions que ce texte n'a fait qu'effleurer pour arriver à la conclusion que la crise dont souffre le travail est durable et que son issue est incertaine.

\section{BIBLIOGRAPHIE}

Accornero, Aris, « Una ricerca sui lavori coordinati e continuativi, fra subordinazione e autonomia ", Lavoro informazione, $\mathrm{n}^{\circ} 22,5,1998$, p.9-14.

Antonmattei, Paul-Henri, Sciberras, Jean-Christophe, « Le travailleur économiquement dépendant : quelle protection ?, Rapport à M. le Ministre du Travail, des Relations sociales, de la Famille et de la Solidarité, novembre 2008. http://travail-emploi.gouv.fr/IMG/pdf/Rapport-AntonmatteiSciberras-07NOV08.pdf. Page consultée le 2 décembre 2008.

Azaïs, Christian, « Dé-segmentation du marché du travail et autonomie », in Sophie Boutillier, Dimitri Uzunidis (dir.), Travailler au $21^{e}$ siècle, Nouveaux modes d'organisation industrielle et spatiale du travail, Collection « Économie, Société, Région », De Boeck Université, 2006, p. 113-127.

Azaïs, Christian, « Labour and Employment in a Globalising World », in Christian Azaïs (dir.), Labour and Employment in a Globalising World : Autonomy, collectives and political dilemmas, Bruxelles, P.I.E. Peter Lang, 2010a, p. 9-27.

Azaïs, Christian, « Subordination or Autonomy? The hybridization of the labour market -The Italian case », in Christian Azaïs (dir.), Labour and Employment in a Globalising World: Autonomy, collectives and political dilemmas, Bruxelles, P.I.E. Peter Lang, 2010b, p. 163-181.

Azaïs, Christian, « Pilotos de helicópteros em São Paulo: o assalariamento entre 'céu aberto' e 'nevoeiro' », Sociologias, UFRGS, Porto Alegre, n² 25, oct.-nov.-déc. 2010c., p. 102-124.

Azaïs Christian, Steck Jean-Fabien, « Éditorial », Espaces et sociétés, 3/2010d, n¹43, p. 7-12.

Beck, Ülrich, The Brave New World of Work, Oxford, Polity Press, 2000.

Bidet, Jacques, Texier, Jacques (dir.), « Le travail fait époque », in La crise du travail, Paris, PUF, Actuel Marx, 1994.

Boyer, Robert, La flexibilité du travail en Europe - une étude comparative des transformations du rapport salarial dans sept pays de 1973 à 1985, Paris, La Découverte, 1987. 
Cabanes, Robert, Georges Isabel, 2014, « Les années Lula : politiques sociales ou néolibéralisme?», Brésil(s), Paris, n 6, 2014.

Cappellin, Paola, Giffoni, Raquel, « As Empresas em Sociedades Contemporâneas: A Responsabilidade Social no Norte e no Sul », Caderno CRH, vol. 28, n 51, sept.-déc. 2007, p. 419-434.

Commission européenne, Base de données AMECO, 2010. http://ec.europa.eu/economy_finance/ db_indicators/ameco/index_en.htm. Page consultée le 15 mars 2015.

Destremau Blandine, Georges Isabel (dir.), Gouverner les pauvres en Amérique latine. Gérer les femmes par l'assistance, Paris, Aix-en-Provence, Karthala - Cherpa, coll. Questions transnationales, à paraître en 2015.

Dobb, Maurice, Wages. A comprehensive study of wage problems, $4^{\mathrm{e}}$ éd., Cambridge, Digswell Place James Nisber \& Co. Ltd (At the University Press), The Cambridge Economic Handbooks, 1955.

Eckert, Henri, «Centralité du travail ou centralité du salariat? », in Paul Bouffartigue, Henri Eckert (dir.), Jean-René Pendaries, Le travail à l'épreuve du salariat - à propos de la fin du travail, Paris, L'Harmattan, Logiques sociales, 1997, p. 51-71.

Giraud, Olivier, « A globalização vista do Norte e do Sul: Quais os seus mecanismos sociais? », Caderno CRH, Salvador, vol. 20, n 51, sept.-déc. 2007, p. 389-399.

Giraud, Olivier, « Les défis de la comparaison à l'âge de la globalisation : pour une approche centrée sur les cas les plus différents inspirée de Clifford Geertz », Critique internationale, Presses de Sciences Politique, $n^{\circ}$ 57, 2012/4, , p. 89-110.

Guitton, Christophe ; Maruani, Margaret ; Reynaud, Emmanuelle (dir.), L'emploi en Espagne. Marché du travail et relations professionnelles, Paris, Syros, 1991.

Hatzfeld, Hélène ; Hatzfeld, Marc ; Ringart, Nadja, Quand la marge est créatrice. Les interstices urbains initiateurs, Paris, Éditions de L'Aube, Coll. Territoires, 1998.

Held, David, McGrew, Anthony (dir.), The global transformations reader -an introduction to the globalisation debate, Cambridge, Polity Press, 2000.

Hoje em Dia, « Pesquisa IBGE aponta melhoria na distribuição de renda no Brasil », Hoje em Dia, 29 septembre 2013. http://www.hojeemdia.com.br/noticias/pesquisa-ibge-aponta-melhoria-nadistribuic-o-de-renda-no-brasil-1.175011. Page consultée le 11 décembre 2013.

INSEE, Partage de la valeur ajoutée, partage des profits et écarts des rémunérations en France, Rapport au Président de la République, 13 mai, Mission présidée par Jean-Philippe Cotis, 2009. www.insee.fr/fr/publications.../rapport_partage_VA.pdf. Page consultée le 10 novembre 2011.

Lacour, Claude, «La tectonique des territoires : d'une métaphore à une théorisation », in Bernard Pecqueur (dir.) Dynamiques territoriales et mutations économiques, Paris, L'Harmattan, 1996, p. 25-48. Lautier, Bruno ; de Miras, Claude ; Morice, Alain, L'État et l'informel, Paris, L'Harmattan, 1991. Maruani, Margaret ; Reynaud, Emmanuelle ; Romani, Claudine (dir.), La flexibilité en Italie, Paris, Syros Alternatives, 1989.

Méda, Dominique, Le travail, une valeur en voie de disparition?, Paris, Aubier/Alto, 1995.

PNAD, Pesquisa nacional de amostragem por domicílios, 2013. http://www.ibge.gov.br/home/ estatistica/populacao/trabalhoerendimento/pnad2013/. Page consultée le 12 février 2015.

Rifkin, Jeremy, La fin du travail, Paris, La Découverte, 1996. 
Supiot, Alain, « Les nouveaux visages de la subordination », Droit social, février 2000, p. 129-145. Thompson, Edward P., « Temps, travail et capitalisme », Libre n 5, p. 3-63, 1979, traduction de l'article paru dans Past and Present, Oxford University Press, $n^{\circ} 38,1967$, p. 56-97.

Thompson, Edward P., La formation de la classe ouvrière anglaise,[éd.orig. The making of the english working class, Londres, Pelican Books], Paris, Le Seuil, trad. de l'anglais par Gilles Dauvé, Mireille Golaszewski, Marie-Noëlle Thibault, 1988 [1963].

\section{NOTES}

1. Cet article est le fruit d'une communication présentée en novembre 2009 « La société salariale en crise. Et le travail dans tout cela?", au colloque annuel de l'Institut des Amériques «Les Amériques face à la crise ».

2. Voir Eckert (1997 : 25).

3. Les ouvrages sur la flexibilité ont foisonné dès la fin des années 1980 (Boyer, 1987 ; Maruani et al., 1989 ; Guitton et al., 1991), à propos de l'Europe, de l'Italie et de l'Espagne, respectivement.

4. Je dois à Donna Kesselman cette expression plus heureuse que les traductions : " globalisation en marche » ou « globalisation en cours ».

5. Terme pris dans le sens de Edward $\mathrm{P}$. Thompson pour qui l'expérience est à l'origine de la construction identitaire et de la «fabrication » (making) de classe. Le terme «fabrication » rend davantage compte des atermoiements, du brouillage et de l'enchevêtrement de situations, ce que je traduirais par hybridation. Voir Thompson (1988).

6. Cette dichotomie est de plus en plus fréquemment remise en cause dans de nombreux domaines. Voir Azaïs et Steck (2010).

7. Ces données ne prennent pas en compte les revenus issus de la sphère financière, qui accentueraient le phénomène.

8. Voir Hoje em Dia (2013).

9. «The unintended consequence of the neoliberal free-market utopia is a Brazilianization of the West [...]. As new developments show in the so-called highly developed economies, this nomadic 'multi-activity' - until now mainly a feature of female labour in the West - is not a premodern relic but a rapidly spreading variant in the late work-societies, where attractive, highly skilled and well-paid full-time employment in on its way out ». Voir Beck (2000:1-2).

10. L'idée répandue dans les années 1960-1970 en Amérique latine que les activités informelles disparaîtraient et seraient absorbées par l'économie capitaliste s'est avérée fausse. C'était supposer que les relations capitalistes marchandes allaient investir l'ensemble des activités économiques, ce qui rappelle le «something will turn up " à propos du «casual » employment. Voir Dobb (1955 : 146).

11. "People have sometimes said of theories of wages that the labour-market is in fact much too complex a tangle of interacting forces for any simplified theory to yield calculations which have much validity outside a special type of situation or a limited period of time ». Voir Dobb (1955: 89).

12. La décision de plusieurs entreprises de s'affranchir de législations du travail contraignantes n'est qu'un exemple parmi d'autres de la pression exercée par les entreprises pour déroger à un droit du travail, construit au fil des années, grâce à la pression des syndicats et aux luttes des travailleurs. Par ailleurs, les baisses de salaires, qui en sont une autre expression, semblent s'installer comme une tendance dure dans les pays développés.

13. Le numéro dirigé par Cabanes R. et Georges I. offre une série d'analyses éclairantes sur l'évolution des politiques sociales depuis l'arrivée du Président Luís Inácio Lula da Silva au Brésil 
et sur la manière dont elles se sont coulées dans le moule du néolibéralisme ambiant. Voir Cabanes et Georges (2014).

\section{RÉSUMÉS}

La crise du travail est analysée à travers deux processus, l'uniformisation et la différenciation, caractéristiques d'une globalisation dynamique. Elle se manifeste par le brouillage des frontières du salariat, au Nord et au Sud, phénomène que traduit le concept d'hybridation, reflet de l'enchevêtrement des formes de mise au travail et de contrats de travail et ce, parfois, pour un même individu. La tendance à la flexibilisation et à la précarisation des travailleurs n'est plus l'apanage des travailleurs du Sud, elle concerne aussi des travailleurs qualifiés. Ce mouvement général s'accompagne de l'émergence d'une zone grise du salariat, zone interstitielle qui rend caduques les dichotomies travail formel/travail informel ; travail/hors-travail ; qualification/ protection sociale, sonnant comme une invitation pour les chercheurs à s'interroger sur la pertinence des catégories. Cette évolution est caractéristique d'un globalising world.

Aujourd'hui, les garanties assorties à l'exercice d'une profession, les droits sociaux, la protection sociale se trouvent affectés et demandent un autre type d'analyse du travail et du salariat, car la forme ethnocentrique provenant des pays développés est devenue inadéquate. Peut-être plus que jamais est-il devenu difficile de distinguer les contours du salariat et de délimiter les situations de dépendance de celles d'autonomie au travail, signe que les frontières du salariat ont tendance à s'élargir et à se décloisonner. Un vaste chantier s'ouvre. Autant de questions que ce texte a tenté d'aborder pour arriver à la conclusion que la crise dont souffre le travail n'est pas passagère ; elle est durable et son issue est incertaine.

The labour crisis is analysed through two processes, standardisation and differentiation, the main characteristics of a globalising world. This can be appreciated through the blurring borders of wage earners, in both Northern and Southern societies, phenomenon which is taken into account through the concept of hybridisation. It reflects the entanglement of various forms of insertion of individuals into the labour market, occurring even for a single individual. The tendency towards workers' flexibilisation and precarisation is no longer the characteristic attribute of workers from the South: even the highly qualified, from South to North, are concerned. This general movement comes along with the emergence of a grey zone surrounding the employment relationship, an in-between zone that makes obsolete traditional dichotomies such as formal/ informal labour; worker/unemployed; qualification/social protection, acting as an invitation for researchers to dwell upon the pertinence of categories. Such an evolution is characteristic of what we have characterised as a globalising world.

Nowadays, the guaranties linked to the exercise of a profession, social rights, social protection are being affected and require a new analysis of labour and wage earning, because the ethnocentric form inherited from developed countries has become blatantly inadequate. Possibly more than ever, it has become difficult to distinguish the contours of employment and situations of dependency from those of autonomy at work, a sign that the borders of wage-earning societies tend to enlarge and to decompartmentalize, opening vast perspectives for research. These are some of the issues this text deals with to reach the conclusion that the crisis currently affecting labour is not cyclical; it is a durable one and any foreseeable solution is uncertain. 
Se analiza la crisis del trabajo a través de dos procesos, la uniformización y la diferenciación, características de una globalización dinámica. Ésta se manifiesta por el borrar de las fronteras del asalariado, en el Norte y en el Sur, fenómeno que traduce el concepto de hibridación, reflejo del entrelazamiento de las formas de inserción en el trabajo y de contratos de trabajo, esto ocurriendo a veces para un mismo indivíduo. La tendencia a la flexibilización y a la precarización de los trabajadores ya no es más lo propio de los trabajadores del Sur, atinge también a trabajadores calificados. Este movimiento general lo acompaña la emergencia de una zona gris del salariado, zona intersticial que vuelve caducas las dicotomías "trabajo formal/trabajo informal"; "trabajo/extra-trabajo" ; calificación/protección social" e incitan a los investigadores a replantear la pertinencia de estas categorías. Esta evolución caracteriza el globalising world.

Hoy, las garantías asociadas al ejercicio de una profesión, los derechos sociales, la protección social están afectados y requieren otro tipo de análisis del trabajo y del salariado, pues la forma etnocéntrica proveniente de los países desarrollados resulta inadecuada. Quizás, más que nunca, resulta difícil delimitar los contornos del salariado e distinguir las situaciones de dependencia de las de autonomía en el trabajo, señal de que las fronteras del salariado tienden a extenderse, incluso hasta evanecerse. Está abriéndose una amplia obra. Este texto ha intentado plantear estas cuestiones para llegar a la conclusión de que la crisis de la que padece el trabajo no es pasajera; es duradera y su salida es incierta.

INDEX

Mots-clés : crise, travail, globalisation, salariat, hybridation, France, Brésil

Keywords : crisis, labour, globalisation, wage-earning, hybridisation, France, Brazil

Palabras claves : crisis, trabajo, globalización, salariado, hibridación, Francia, Brasil

\section{AUTEUR}

\section{CHRISTIAN AZAÏS}

Christian Azaïs est socio-économiste, enseignant à Amiens et chercheur au Laboratoire interdisciplinaire de sociologie économique (LISE) - UMR 3320, CNAM/Paris.Professeur de science politique à l'Université fédérale de la Paraíba (Brésil), de 1982 à 1994, il mène depuis ce temps des recherches sur le travail, en Amérique latine et en France, principalement. Actuellement engagé dans le programme ANR Zogris, portant sur l'analyse comparative de la transformation des normes d'emploi - zones grises -, il s'intéresse à la transformation des normes d'emploi et de travail au Brésil et au Mexique à partir d'une recherche comparative sur les pilotes d'hélicoptère à São Paulo et Mexico. Cette recherche a fait suite à celle menée dans le cadre du programme ANR Metraljeux qui analysait les logiques institutionnelles et le jeu des acteurs dans quatre métropoles latino-américaines, Buenos Aires, Caracas, Mexico et São Paulo. 